'Departamento de Nefrología, Clínica Dávila, Santiago, Chile. ${ }^{2}$ Facultad de Medicina, Universidad de Los Andes, Santiago, Chile.

${ }^{3}$ Departamento de

Anatomía Patológica,

Clínica Dávila, Santiago, Chile.

${ }^{4}$ Departamento de Radiología, Clínica Dávila, Santiago, Chile.

aEstudiante de Medicina, Universidad de Los Andes, Santiago, Chile.

Recibido el 23 de noviembre de 2012 , aceptado el 19 de abril de 2013.

Correspondencia a: Dr. Antonio Vukusich Covacic

Recoleta 464, Recoleta, Santiago.

Teléfono: 27308021

E-mail: avukusic@davila.cl

\section{Biopsia renal en el segundo trimestre del embarazo. Dos casos clínicos}

\author{
ANTONIO VUKUSICH ${ }^{1,2}$, GONZALO VALLS ${ }^{1}$, \\ MARGARITA ENBERG ${ }^{2}$, MARÍA JOSÉ DE LA PIEDRAa, \\ LUIS CONTRERAS ${ }^{3}$, JUAN CARLOS BRAVO ${ }^{4}$
}

\section{Renal biopsies during the second trimester of pregnancy. Report of two cases}

Kidney biopsies were performed in two women during their 21 th and 24th week of pregnancy. The first patient developed an abrupt nephrotic syndrome without hypertension or kidney failure. The pathological study disclosed diffuse podocyte alterations such as those observed in focal and segmental glomerulosclerosis, which had a good response to steroidal treatment. The second patient had a progressive renal failure associated with non-nephrotic proteinuria. The biopsy disclosed a fibrillary glomerulopathy.

(Rev Med Chile 2013; 141: 1072-1075).

Key words: Acute kidney injury; Biopsy, needle; Glomerulosclerosis focal segmental; Nephrotic syndrome; Pregnancy.
L as glomerulopatías primarias que se inician en el embarazo son muy infrecuentes. Aquellas que cursan con síndrome nefrótico (SN) tienen una incidencia de 0,12 a $0,25 \times 1 \cdot 000^{1,2}$. Existen diferentes opiniones sobre la necesidad de una biopsia renal durante la gestación. Los hallazgos histológicos reportados incluyen nefritis lúpica, nefropatía de cambios mínimos, glomeruloesclerosis focal y segmentaria (GEFS), glomerulonefritis mesangioproliferativa y nefropatía por IgA $\mathrm{A}^{3}$. Se han reportado casos de $\mathrm{SN}$ asociado a tumor trofoblástico con diversas lesiones glomerulares incluyendo la glomerulopatía membranosa, y se ha sugerido, que las anormalidades fetales o placentarias deben ser excluidas antes de plantear la biopsia ${ }^{2,4,5}$. Comunicamos un caso de $\mathrm{SN}$ y otro de falla renal progresiva durante el segundo trimestre del embarazo en los que se decidió realizar una biopsia renal.

\section{Paciente $N^{o} 1$}

Primigesta de 21 años, sin antecedentes. Cursando un embarazo de 21 semanas, desarrolló en forma brusca edema generalizado sin hipertensión arterial (HTA). Fue hospitalizada y se comprobó un $\mathrm{SN}$ con proteinuria de $14 \mathrm{~g}$ /día, sin deterioro funcional renal (creatininemia $0,6 \mathrm{mg} / \mathrm{dL}$ ). El hemograma, la glicemia y el test de tolerancia a la glucosa oral resultaron normales. Colesterol total $387 \mathrm{mg} / \mathrm{dL}$. El sedimento urinario mostró 15-20 glóbulos rojos por campo. Los anticuerpos antinucleares (ANA), contra el citoplasma de neutrófilos (ANCA), anti ADN, anticardiolipinas, anticoagulante lúpico; complemento C3 y C4, VDRL, serología para VIH, hepatitis $\mathrm{B}$ y C resultaron todos negativos. Ecotomografía: riñones de aspecto y tamaño normal. Inició reposo, restricción de sal y furosemida. Mantuvo proteinuria masiva sin fundir edemas. Frente a la gravedad del cuadro (albuminemia 1,2 g/dL; proteinemia 3,2 g/dL), los riesgos potenciales de una terapia esteroidal empírica y los casi cinco meses restantes para el término del embarazo, se decidió practicar, previo consentimiento informado, una biopsia renal.

\section{Paciente No 2}

Multípara de 34 años con antecedentes de enfermedad de Crohn inactiva. Cursando un embarazo de 24 semanas consultó por dolor epigástrico. 
No presentaba HTA ni edema. Laboratorio: proteinuria de 1,2 g/día, micro hematuria sin dismorfia, creatininemia: 1,4 mg/dL. Hemograma, glicemia, ANA, ANCA, anti DNA, C3 y C4, electroforesis de proteínas plasmáticas, inmunofijación en plasma y orina, serología para VIH, hepatitis C y B resultaron normales. Ecotomografía: estructura y tamaño renal conservados. Por deterioro progresivo de la función renal (alza de creatininemia a $1,7 \mathrm{mg} / \mathrm{dL}$ en una semana), se realizó una biopsia renal previo consentimiento informado.

\section{Procedimiento}

Ambas biopsias se realizaron en decúbito prono, bajo anestesia local y guía ecográfica (ATL 3000, transductor convexo 3-5 megaHertz), por un radiólogo intervencionista. Se puncionó el polo inferior de cada riñón izquierdo con aguja coaxial de $17 \mathrm{~g} \mathrm{x} 10 \mathrm{~cm}$. A través de ella se introdujo una pistola de biopsia automática percutánea (biopince, ATM) de $18 \mathrm{~g} \mathrm{x} 15 \mathrm{~cm}$. Se obtuvieron dos cilindros de tejido manteniendo siempre la aguja coaxial como punto fijo en situación intrarrenal. El patólogo, presente durante el procedimiento, comprobó la suficiencia de las muestras. No se presentaron complicaciones.

En la primera paciente se obtuvieron 29 glomérulos 2 de ellos globalmente esclerosados y 1 con esclerosis segmentaria de 25\% y adherencias a la cápsula de Bowman. Inmunofluorescencia
(IF): presencia granular focal de IgM. Microscopia electrónica (ME): fusión pedicelar extensa con cambios degenerativos a nivel podocitario. Conclusión: enfermedad podocitaria difusa tipo GEFS variante clásica (Figura 1). Inició prednisona $60 \mathrm{mg} /$ día. Proteinuria desapareció en cuatro semanas. No presentó HTA, complicaciones de la terapia esteroidal, ni falla renal. Parto vaginal a las 38 semanas de gestación. Recién nacido $(\mathrm{RN})$ sano de $3.160 \mathrm{~g}$.

En la segunda paciente se obtuvieron 34 glomérulos, 2 de ellos globalmente esclerosados y el resto con arquitectura de aspecto pseudonodular. IF: depósito de IgG con predominio de cadenas livianas lambda en capilares, nódulos y membrana basal tubular. ME: glomérulo con engrosamiento de membranas basales, pérdida de pedicelos en células epiteliales y distorsión pseudonodular con abundantes depósitos mesangiales que adquieren una estructura fibrilar de 20 a 25 nanómetros de diámetro. Rojo de Congo (-). Conclusión: glomerulopatía fibrilar (GF) (Figura 2). Mielograma normal. No se hizo citometría de flujo. Desarrolló HTA. Con metildopa $250 \mathrm{mg}$ c/12 h e hidralazina $50 \mathrm{mg}$ c/8 h se consiguieron cifras de 120/80 mmHg. A las 34 semanas presentó desprendimiento de placenta. Cesárea de emergencia obtuvo un $\mathrm{RN}$ sano de $2.300 \mathrm{~g}$.

Las placentas de las dos pacientes eran morfológicamente normales.

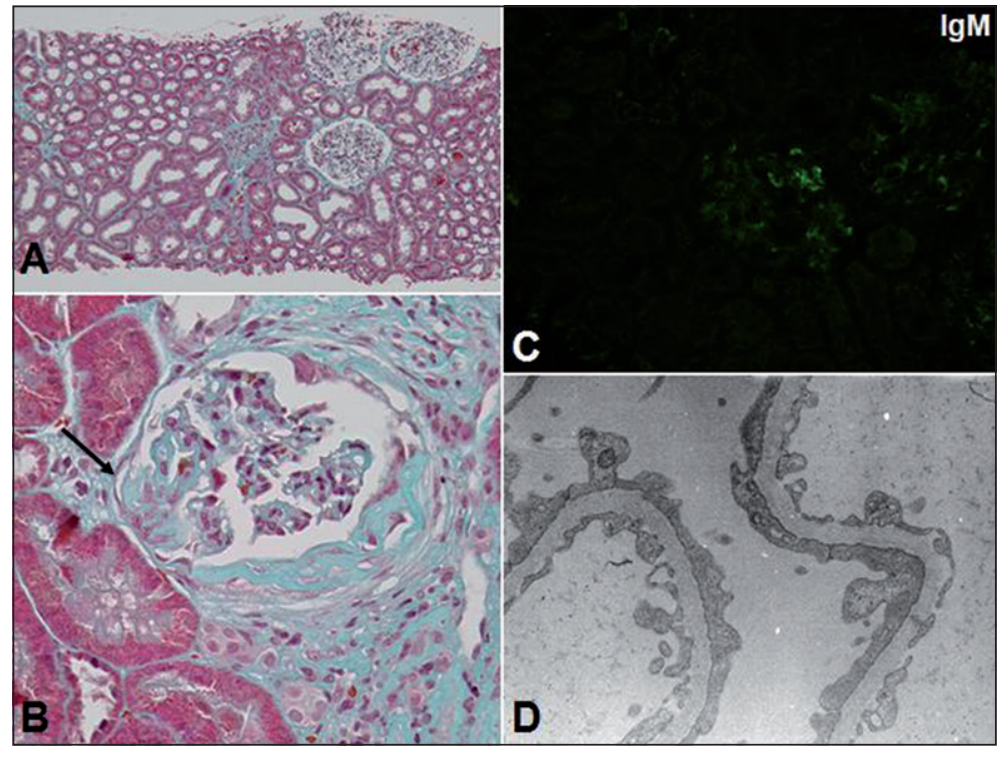

Figura 1. Glomeruloesclerosis focal y segmentaria. 


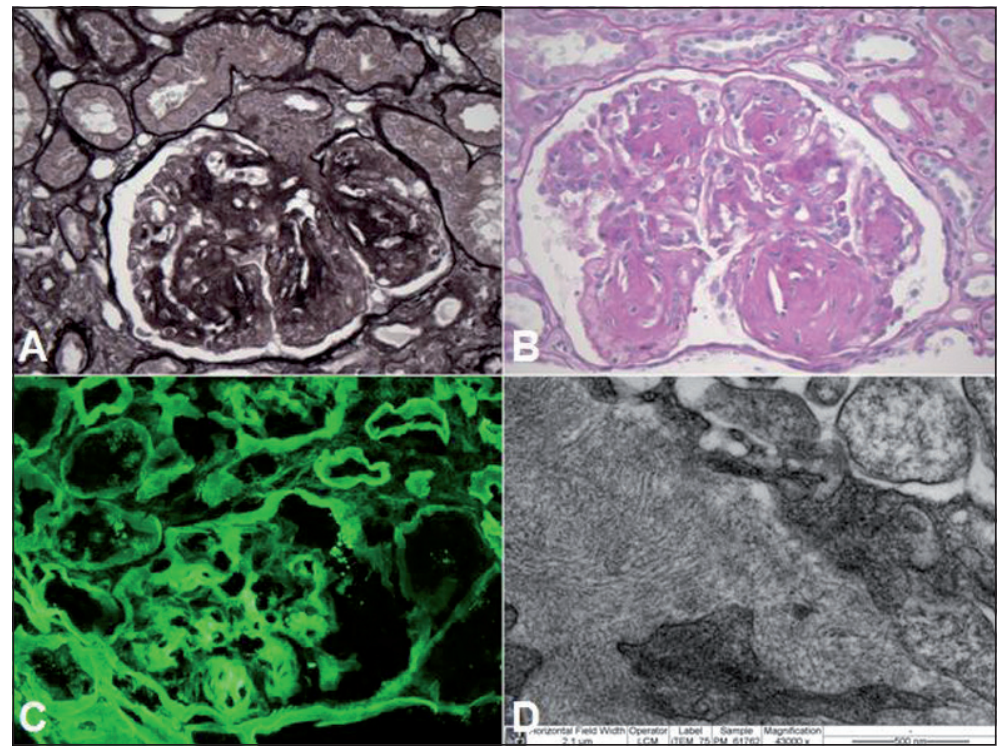

Figura 2. Glomerulonefritis fibrilar con predominio de cadenas livianas lambda.

\section{Discusión}

La proteinuria en el embarazo es frecuente. Se consideran "fisiológicos" valores de hasta $300 \mathrm{mg} /$ día ${ }^{6}$. Una excreción mayor, especialmente durante el primer trimestre, o asociada a HTA o falla renal, sugiere la presencia de enfermedad renal primaria o preeclampsia en el tercer trimestre ${ }^{2}$. Las glomerulapatías que debutan en el embarazo son muy infrecuentes y determinan un mal pronóstico materno y fetal ${ }^{5-9}$. Barceló et al refieren que la mortalidad perinatal en mujeres portadoras de glomerulopatías primarias alcanza $21 \%$, y se elevaría a $31 \%$, si se agrega HTA. También aumentan el riesgo de aborto espontáneo, prematuridad y bajo peso al nacer, eventos que correlacionan con la magnitud de la proteinuria, la hipoalbuminemia y la precocidad con que ambas se presentan durante la gestación ${ }^{10}$.

Frente a esta situación los clínicos tienen pocas opciones:

1. Mantener un tratamiento sindromático hasta el final del embarazo y luego efectuar la biopsia, lo que podría empeorar el pronóstico.

2. Iniciar esteroides en forma empírica, apostando a la presencia de una patología sensible, lo que puede complicarse con los efectos no deseados de esta terapia.

3. Iniciar el tratamiento o abstenerse de este, según la naturaleza de la enfermedad, para lo cual se debe hacer una biopsia renal, aceptando la relación riesgo beneficio de la misma.

La biopsia de la primera paciente mostró 2 de 29 glomérulos globalmente esclerosados y uno con esclerosis segmentaria sin características de las variedades colapsante ni "tip lesion". Se diagnosticó una enfermedad pedicelar tipo GEFS clásica y respondió a esteroides en cuatro semanas. No había historia familiar de enfermedad renal y no se encontraron elementos para plantear una forma secundaria de GEFS. La segunda paciente no era diabética, no tenía paraproteínas en orina ni en sangre y tenía rojo de Congo negativo lo que descarta una gammapatía monoclonal y amiloidosis. La ME mostró fibrillas que por sus dimensiones son específicas de una GF.

Los primeros reportes de biopsias renales realizados durante el embarazo datan de $1960^{11}$. En esa época, la tecnología de apoyo en imágenes era imprecisa y el material usado para la punción era menos seguro. La tasa de complicaciones era elevada: hematuria macroscópica $(16,7 \%)$, hematoma peri-renal $(4,4 \%)$; incluso un caso de muerte materna asociada al procedimiento ${ }^{12,13}$. Publicaciones posteriores muestran una disminución franca de las complicaciones. En la última década queremos destacar dos por su mayor casuística: una revisión retrospectiva (1990-1999), publicada por Chen et al. ( $\mathrm{n}=15$, mediana de edad 28 años), y otra 
realizada por Day et al que abarca los años 1983$2004(\mathrm{n}=20 \text {, mediana de edad } 29 \text { años })^{14,3}$. Llama la atención el número de casos comunicados que no supera los 15 por década. Dentro de las causas que motivaron la biopsia destacan el SN ( $80 \%$ en la primera serie y $20 \%$ en la segunda) y la falla renal de causa incierta (20\% y $40 \%$ respectivamente). La histología mostró una enfermedad glomerular en $85 \%$ de las biopsias y el resultado produjo un cambio terapéutico inmediato en casi $2 / 3$ de los casos. La mortalidad perinatal fue $1 / 15(6,6 \%)$ y de 4/20 (20\%) en ambas series, respectivamente. Los procedimientos fueron realizados alrededor de la semana 22 de embarazo y bajo guía ecográfica, lo que podría explicar el bajo porcentaje de complicaciones: sólo hematuria macroscópica (2/35 pacientes) que remitió espontáneamente.

Una de nuestras pacientes debutó precozmente con un $\mathrm{SN}$ grave, sus riñones eran de aspecto normal y no tenía HTA, trombocitopenia, o alteraciones de coagulación. La otra desarrolló una falla renal aguda de causa incierta. Ambas reunían las condiciones que se estiman necesarias para una adecuada relación riesgo/beneficio de la biopsia renal ${ }^{3}$.

En nuestra experiencia, cuando el operador es un radiólogo intervencionista se logra un mayor rendimiento de las imágenes de apoyo. Así, en 80\% de las biopsias realizadas en nuestra clínica en los últimos 5 años $(\mathrm{n}=370)$ fueron suficientes 2 punciones por paciente y en menos de $5 \%$ de los casos fue necesario obtener 4 o más cilindros de tejido. El criterio del patólogo es que las muestras son suficientes para un buen diagnóstico si contienen un mínimo de 10 glomérulos para la microscopia de luz, 5 para IF y 1 para ME. En nuestras pacientes, las biopsias establecieron diagnósticos precisos, facilitando un adecuado control de la enfermedad, en el primer caso, y evitando el uso inútil de esteroides en el segundo. No se presentaron complicaciones, sin embargo, siempre son posibles. En la eventualidad de un sangramiento importante, o la sospecha de una fístula arterio-venosa traumática, el mismo operador puede precisar el diagnóstico con imágenes y tratar el problema, sin demora, con técnicas hemostáticas endovasculares.

Finalmente, coincidimos con la mayoría de los autores que señalan que la biopsia renal debe ser considerada durante los primeros 2 trimestres del embarazo sólo en mujeres que presentan SN en ausencia de una enfermedad sistémica que lo explique o que tienen una falla renal de causa incierta. Si el SN o la falla renal se presentan durante el tercer trimestre se recomienda acelerar el parto y hacer la biopsia después.

\section{Referencias}

1. Studd J, Blaney J. Pregnancy and Nephrotic Syndrome. Brit med J 1969; 1: 276-9.

2. Pandya B, Gibson S, Robertson I. Nephrotic syndrome in early pregnancy-is renal biopsy always necessary? Nephrol Dial Transplant 2002; 17: 672-4.

3. Day C, Hewins P, Hildebrand S, Sheikh L, Taylor G, Kilby $\mathrm{M}$, et al. The role of renal biopsy in women with kidney disease identified in pregnancy. Nephrol Dial Transplant 2008; 23: 201-6.

4. Cohen AW, Burton HG. Nephrotic syndrome due to preeclamptic nephropathy in a hydatidiform mole and coexist fetos. Obstet Gynaecol 1979; 53: 130-4.

5. Batra V, Kalra O, Mathur P, Kavita, Dev G. Membranous glomerulopathy associated with placental site trophoblastic tumour: A case report. Nephrol Dial Transplant 2007; 22: 1766-8.

6. Priya A, Schmidt R, Holley J. Pregnancy and Renal Disease. En: Lerma E, Berns J, Nissenson A, Editores, Nephrology and Hypertension. Current Diagnosis and Treatment. New York, Mac Graw Hill Lange; 2009, p. 492.

7. Fischer M, Lehnerz S, Hebert J, Parikh C. Kidney disease is an independent risk factor for adverse fetal and maternal outcomes in pregnancy. Am J Kidney Dis 2004; 43: 415-8.

8. Williams W, Ecker J, Thadani R, Rahemetullah A. Case 38-2005: A 29-Year-Old Pregnant Woman with the Nephrotic Syndrome and Hypertension. N Eng J Med 2005; 353: 2590-600.

9. Jungers P, Chauveau D. Pregnancy in renal disease. Kidney International 1997; 52: 871-85.

10. Barceló P, López-Lillo J, Cabero L, Del Río G. Successful pregnancy in primary glomerular disease. Kidney International 1986; 30: 914-9.

11. Pollak V E and Nettles J B. Kidney in Toxemia of Pregnancy Medicine 1960; 39: 469.

12. Schewitz L, Friedman I, Pollak V. Bleeding after renal biopsy in pregnancy. Obstet Gynecol 1965; 26: 295.

13. Packham D, Fairley K. Renal biopsy: indications and complications in pregnancy. Br J Obstet Gynaecol 1987; 94: 935.

14. Chen H, Lin H, Yeh J, Chen C. Renal biopsy in pregnancies complicated by undetermined renal disease. Acta Obstet Gynecol Scand 2001; 80: 888-92. 